\title{
Applications of an Iterative Wiener-Hopf Method to Aeroacoustics
}

\author{
Matthew J. Priddin*, Anastasia V. Kisil ${ }^{\dagger}$ and Lorna J. Ayton* \\ University of Cambridge, Cambridge, UK CB3 OWA
}

\begin{abstract}
This paper explores an iterative approach to Wiener-Hopf problems arising from mixed boundary value problems associated with fluid-structure interaction. We apply the method to problems in aeroacoustics involving multiple boundary condition junctions that yield matrix Wiener-Hopf problems which are often impossible to solve exactly. By identifying weak physical couplings within the system reliable and rapidly convergent schemes may be constructed. We present an effective implementation utilizing spectral techniques which we hope to be accessible to the broader engineering community. The efficiency of the method facilitates optimization and parameter studies useful for real-world applications. It naturally fits into the established Wiener-Hopf literature, in principle enabling previous applications of the WienerHopf technique to various linear partial differential equations in two and three dimensions to be extended to problems with additional boundary condition junctions.
\end{abstract}

\section{Introduction}

The Wiener-Hopf (WH) technique provides a powerful analytic method for solving mixed boundary problems that arise when considering fluid-structure interactions. Its contributions to aeroacoustic theory include investigations of canonical scattering problems [1-3], jet engine exhaust configurations [4]-8] and most recently quiet aerofoil adaptations inspired by the silent flight of owls: poroelasticity [9-11] and serrations[12, 13]. The application of such material modifications introduces new junctions at which the physical boundary condition to be imposed changes sharply. Indeed, a multiplicity of such junctions is generically present in devices composed of distinct materials. These situations yield matrix Wiener-Hopf problems for which exact solutions are typically impractical, indeed general constructive approaches for exact solutions do not exist.

Useful approximate approaches to achieve the required Wiener-Hopf factorizations include using Padé approximations [7, 10, 14, 15] and asymptotic approximations [1, 16, 17] that may be more easily factorized than the original functions. Unfortunately each has a limited domain of applicability excluding many problems of physical interest. Specifically, the prototypical Wiener-Hopf kernel

$$
\gamma(\alpha)=\sqrt{\alpha^{2}-k^{2}}
$$

does not permit a uniform rational approximation on the real line, limiting the utility of Padé approximation, and asymptotic methods are innately constrained to extreme parameter regimes. Numerical approaches are available in Fredholm factorization[18] used in electromagnetic scattering, and a singular integral equation formulation associated with Riemann-Hilbert problems [19], but each requires more computationally expensive matrix inversions avoided by direct approaches, in addition to precise consideration of singular features to achieve effective implementations.

We instead consider the iterative approach proposed in [11, 20] which provides an effective, rigorous framework to attack matrix Wiener-Hopf problems. By identifying weak couplings within the system, one may reduce the dimension of the Wiener-Hopf system to be initially solved and so construct an iterative scheme based on scalar problems. Convergence to the exact solution is guaranteed for sufficiently high frequencies [20]. We demonstrate that this approach can rapidly produce reliable solutions, even at relatively low frequencies. Our implementation is facilitated by the spectral numerical framework introduced in [19, 21]. This paper intends to clearly outline the framework so far developed and clarify its scope in relation to aeroacoustics.

We proceed as follows: we first introduce the method and discuss its practical implementation. The method is then applied to two problems relevant to aeroacoustics:

A. scattering from a finite plate to review the method

\footnotetext{
*Graduate Student, Department of Applied Mathematics and Theoretical Physics, Student Member AIAA. mjp98@cam.ac.uk

†Sultan Qaboos Research Fellow/College Lecturer in Mathematics, Corpus Christi College. a.kisil@maths.cam.ac.uk

${ }^{\ddagger}$ EPSRC Early Career Fellow, Department of Applied Mathematics and Theoretical Physics. 1.j.ayton@damtp.cam.ac.uk
} 
B. scattering from a set of collinear finite plates to demonstrate applicability to problems with many junctions We close by briefly discussing the potential of the method, emphasising that this technique may be applied to problems involving various linear differential operators in two and three dimensions with boundary conditions imposed on parallel sections.

\section{Method}

This section describes the iterative procedure for solving matrix Wiener-Hopf problems. We first review how a Wiener-Hopf problem may be formulated from the boundary value problem of interest using integral transforms. We then outline how the problem so found may be solved using iteration, and steps towards implementing this efficiently numerically.

\section{A. Generating Wiener-Hopf problems associated with aeroacoustics}

To ground our discussion we consider specific example: scattering of an incident disturbance $\phi_{i}$ by a plate of finite length and infinite span. A schematic diagram is presented in figure 1 and results will be presented later in section III.A

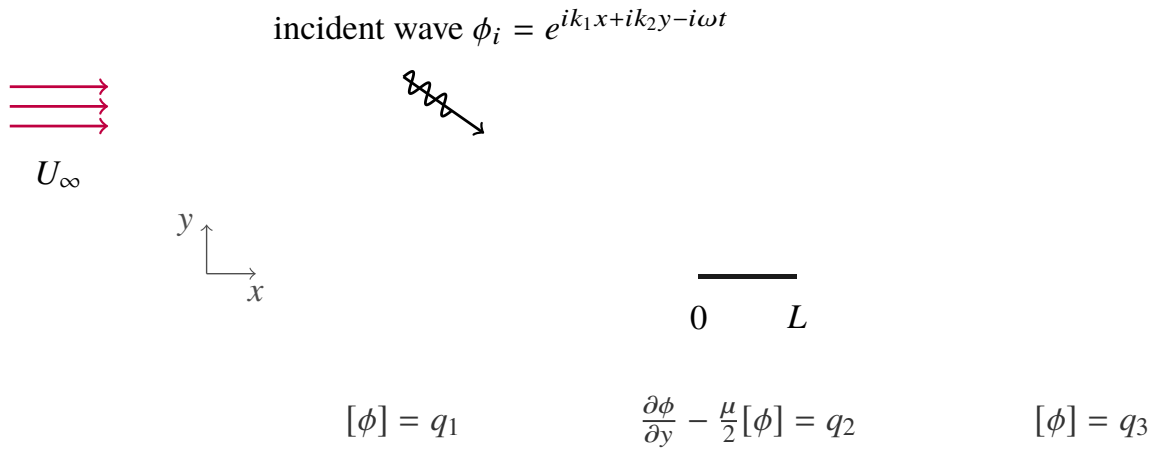

Fig. 1 Scattering from a finite plate. We consider a prescribed incident gust $\phi_{i}$ with interacting with a finite plate of length $L$ on which a Robin boundary condition is imposed. This formulation can model a rigid, porous or poroelastic plate.

Many aeroacoustic scattering problems such as that depicted in figure 1 reduce to solving the 2D Helmholtz equation

$$
\left[\nabla^{2}+w^{2}\right] \phi=0
$$

for $\phi$, the scattered part of an acoustic field. This might variously be the pressure, velocity potential or a generalised acoustic potential [22]. For instance, for uniform background flow the convective wave equation may be reduced to this form using a convective transform.

Boundary conditions A boundary value problem is given by imposing inhomogeneous boundary conditions provided by the geometry and incident disturbance. We consider a finite plate of length $L$ at $y=0$ on which a Robin boundary condition is imposed to model, for example, permeability of the plate. We impose continuity of the field elsewhere:

$$
\begin{array}{rrrr}
{[\phi(x, 0)]_{-}^{+}=q_{1}(x)} & \text { upstream } & -\infty<x<0 \\
\frac{\partial \phi}{\partial y}(x, 0)-\frac{\mu}{2}[\phi(x, 0)]_{-}^{+}=q_{2}(x) & \text { porous } & 0<x<L \\
{[\phi(x, 0)]_{-}^{+}=q_{3}(x)} & \text { downstream } & L<x
\end{array}
$$

We additionally require $\phi^{\prime}=\frac{\partial \phi}{\partial y}$ to be continuous on $y=0$. The parameter $\mu$ in the Robin boundary condition might model the porosity of the plate and is taken to be constant. The final conditions to be imposed are the Sommerfeld radiation condition for outgoing waves at infinity: $\sqrt{x^{2}+y^{2}}=r \rightarrow \infty$ :

$$
r^{-1 / 2}\left[\frac{\partial}{\partial r}-i w\right] \phi \rightarrow 0, \quad r \rightarrow \infty
$$


and that we seek the least singular solution at the edges [1].

Formulation of a spectral (Wiener-Hopf) equation A Wiener-Hopf problem is now found by Fourier transforming the governing equation (2) and boundary conditions (3). We define the $x$-Fourier transform along an interval of the real line $[a, b]$ by

$$
\Phi_{[a, b]}(\alpha, \cdot)=\int_{[a, b]} \phi(x, \cdot) e^{i \alpha x} \mathrm{~d} x
$$

and introduce the notation $(-\infty, a]=[-, a],[a, \infty)=[a,+]$ and $\Phi=\Phi_{(-\infty, \infty)}$. Solving the resulting ordinary differential equation and imposing the far-field radiation condition gives

$$
\Phi(\alpha, y)= \begin{cases}A(\alpha) e^{-\gamma(\alpha) y} & y>0 \\ B(\alpha) e^{+\gamma(\alpha) y} & y<0\end{cases}
$$

where $\gamma(\alpha)=\sqrt{\alpha^{2}-w^{2}}$ with branch cuts chosen to be the rays $\pm w+e^{ \pm i \pi / 2}[0, \infty]$. From this known form of $\Phi$ and required continuity of $\phi$ it follows that $\Phi$ is anti-symmetric in $y$. Restricting attention to $y>0$, so $\Phi(\alpha, 0) \equiv \Phi(\alpha, 0+)$, the boundary conditions on $\Phi$ reduce to

$$
\begin{aligned}
\Phi_{[-, 0]} & =Q_{1} \\
\Phi_{[0, L]}^{\prime}-\mu \Phi_{[0, L]} & =Q_{2} \\
\Phi_{[L,+]} & =Q_{3}
\end{aligned}
$$

The known form of $\Phi$ in (6) provides the relation $\Phi^{\prime}+\gamma \Phi=0$ for $y>0$. Substituting the boundary conditions (7) into this relation yields

$$
\Phi_{[-, 0]}^{\prime}+Q_{2}+\Phi_{[L,+]}^{\prime}+\gamma Q_{1}+(\gamma+\mu) \Phi_{[0, L]}+\gamma Q_{3}=0
$$

an equation in the spectral variable $\alpha$ relating partial Fourier transforms of unknown boundary values. This forms the basis of our Wiener-Hopf system.

\section{B. Solving Wiener-Hopf problems by iteration}

For many problems similar to that considered in the previous section, we generically find a matrix Wiener-Hopf system of the form

$$
A \Phi+B \Psi=F
$$

where $A$ and $B$ are matrices and $\Phi, \Psi$ (unknown, except certain analyticity properties) and $F$ (known) are vectors. Our approach considers this system as a set of coupled scalar problems:

$$
A_{n n} \Phi_{n}+B_{n n} \Psi_{n}=F_{n}-\sum_{m \neq n}\left[A_{n m} \Phi_{m}+B_{n m} \Psi_{m}\right]
$$

We seek to construct a convergent fixed-point iterative scheme by formulating the system such that the couplings are weak. An appropriate choice may often be informed by physical considerations to give

$$
A_{n n} \Phi_{n}+B_{n n} \Psi_{n}=F_{n}+\varepsilon_{n}(\Phi, \Psi)
$$

where $\varepsilon$ represents a sub-dominant coupling. The scheme may be initialised by providing an initial estimate of the terms on the right hand side, and thenceforth use the last computed value for the quantity. We note that for large systems there may be a large degree of freedom when choosing the sequence in which to solve the scalar problems; we will return to this question in section III.B.

Example: Finite plate For scattering problems we anticipate a weak coupling to be provided by 'backscattering' between junctions. We so rewrite the spectral equation (8) to sequentially focus on the junctions at $x=0$ and $x=L$. First introducing plus/minus splitfunctions $\Phi_{ \pm}$analytic in the upper/lower half planes with at worst algebraic decay as $|\alpha| \rightarrow \infty$ in their respective half-plane of analyticity:

$$
\Phi_{-}^{\prime(0)}=\Phi_{[-, 0]}, \quad \Phi_{+}^{(0)}=\Phi_{[0, L]}, \quad \Phi_{-}^{(L)}=e^{-i \alpha L} \Phi_{[0, L]}, \quad \Phi_{+}^{\prime(L)}=e^{-i \alpha L} \Phi_{[L,+]},
$$


equation $(8)$ becomes

$$
\begin{aligned}
& (\gamma+\mu) \Phi_{+}^{(0)}+\Phi_{-}^{\prime(0)}=-e^{i \alpha L}{\Phi_{+}^{\prime(L)}}^{(L)} F^{(0)} \\
& (\gamma+\mu) \Phi_{-}^{(L)}+\Phi_{+}^{\prime(L)}=-e^{-i \alpha L} \Phi_{-}^{\prime(0)}+F^{(L)}
\end{aligned}
$$

where the known forcing terms are given by $F^{(0)}=-\gamma Q_{3}-Q_{2}-\gamma Q_{1}$ and $F^{(L)}=e^{-i \alpha L} F^{(0)}$.

An iterative fixed-point scheme can be constructed by solving, at iteration $m$, the scalar Wiener-Hopf problems

$$
\begin{aligned}
& (\gamma+\mu) \Phi_{+}^{(0) m}+\Phi_{-}^{\prime(0) m}=-e^{i \alpha L}{\Phi_{+}^{\prime(L) m-1}}^{(0)}+F^{(0)} \\
& (\gamma+\mu) \Phi_{-}^{(L) m}+\Phi_{+}^{\prime(L) m}=-e^{-i \alpha L} \Phi_{-}^{\prime(0) m-1}+F^{(L)}
\end{aligned}
$$

The scheme may be initialised for $n=0$ by setting quantities with negative index to zero.

Recovering physical values of interest The scattered velocity potential $\phi$ may be recovered by inverting the spatial Fourier transform:

$$
\phi(x, y)=\frac{\operatorname{sgn} y}{2 \pi} \int_{-\infty}^{\infty} \Phi(\alpha, y) e^{-|y| \gamma(\alpha)-i \alpha x} \mathrm{~d} \alpha
$$

This may be evaluated efficiently numerically by deforming the contour to a path of steepest descent, and an asymptotic steepest descent approximation can yield accurate far-field approximations:

$$
\phi(r, \theta) \sim \operatorname{sgn} y \frac{\sqrt{w} e^{i \pi / 4}}{\sqrt{2 \pi}} \Phi(-w \cos \theta, 0) \sin \theta \frac{e^{i w r}}{\sqrt{r}}
$$

As in [11] we define the far-field directivity $D(\theta)$ by

$$
\phi(x, y) \sim D(\theta) \frac{e^{i w r}}{\sqrt{r}}
$$

\section{Numerical implementation: solution of scalar Wiener-Hopf problems}

We now consider how to solve the scalar Wiener-Hopf problems systematically on a computer. The standard mathematical theory is presented in [1], which we must first partially review.

Central to the Wiener-Hopf technique is the rearrangement of an equation into parts analytic in overlapping regions $\mathcal{R}^{ \pm}$(typically deformations of the upper/lower half-planes $\operatorname{Im} z \gtrless 0$ ) that together cover the complex plane. This first requires a multiplicative factorization of the premultiplying factor, or kernel: e.g. $(\gamma+\mu)$ in equation (14). Having used this to isolate the analyticity of terms involving unknown functions, we require additive factorizations of the remaining terms: e.g. those arising from the terms on the LHS of equation (14). Such additive factorizations (and thereby also scalar multiplicative factorizations) may be generically obtained by performing Cauchy transforms [1]. We define the Cauchy transform $C_{\Gamma}[\phi]$ over a contour $\Gamma$ for $\alpha \notin \Gamma$ of a function $\phi(x, \cdot)$ via

$$
\mathcal{C}_{\Gamma}[\phi](\alpha, \cdot)=\frac{1}{2 \pi i} \int_{\Gamma} \frac{\phi(x, \cdot)}{x-\alpha} \mathrm{d} x
$$

The iterative approach solves a sequence of scalar problems and so requires the repeated computation of the associated integrals.

To efficiently and accurately compute Cauchy transforms numerically we follow [19]. We first uniformly represent the function along the transform contour $\Gamma$ in a basis that encodes singular features, then employ known analytic expressions for the transforms of these basis functions. In this way cancellation errors associated with singular integrals may be largely avoided. For our purposes it is sufficient to consider contours formed of intervals and rays. Cauchy transforms along arbitrary intervals and rays may be related to Cauchy transforms along the unit interval $[-1,1]$ by Möbius mappings.

In order to obtain spectral convergence it is necessary to carefully encode endpoint singularities, which for the problems we consider look like $x^{-1 / 2}$. In this case Cauchy transforms may be expressed simply, avoiding the need to evaluate special functions. We first expand in the so-called vanishing basis [19] of Chebyshev polynomials of the first kind, given by

$$
T_{0}^{z}(x)=1, \quad T_{1}^{z}(x)=x, \quad T_{n}^{z}(x)=T_{n}(x)-T_{n-2}(x), \quad \text { for } n \geq 2
$$


weighted by $(1-x)^{-0.5}(1+x)^{-0.5}$. Then for $n \geq 2$ we have

$$
\left.C_{\Gamma}\left[T_{n}^{z}(x)(1-x)^{-0.5}(1+x)^{-0.5}\right)\right](z)=i\left(J_{+}^{-1}(z)\right)^{n-1}
$$

where $J_{+}^{-1}=z-\sqrt{z-1} \sqrt{z+1}$ is one of the right inverses of the Joukowsky map. In general we require Cauchy transforms of functions with other endpoint behaviours; algebraic endpoint singularities may be considered by expanding

in Jacobi polynomials $P_{n}^{(a, b)}$, whose Cauchy transforms may be represented in terms of the Gauss hypergeometric function.

The method has been implemented in MatLab and Julia employing the approximation packages CheBfun and ApproxFun respectively. The moments of the Cauchy transforms evaluated at points of interest may be precomputed, decoupling this computation expense from the iterative procedure. For problems so far considered typically fewer than 100 degrees of freedom are sufficient in order to achieve machine accuracy. Each factorization is fast, taken less than a millisecond when provided kernel values.

\section{Results}

This section explores the iterative Wiener-Hopf method described above by applying it to two problems relevant to aeroacoustics.

\section{A. Finite plate}

We first consider scattering from a finite rigid plate. This model problem was pictured in figure 1 and the derivation of the associated Wiener-Hopf equations was presented in section II.B.

Validation We first validate the present implementation by considering scattering from a finite plate. This canonical problem has been investigated by numerous approaches including an approximate Wiener-Hopf technique [1], the iterative matrix Wiener-Hopf technique[11], expansions in Mathieu functions or Chebyshev polynomials [11, 23, 24], the Unified Transform method [25]. In figure 2a we observe good agreement with the the exact solution given in terms of Mathieu functions presented in [11].

Convergence In figure $2 \mathrm{a}$ we observe that the the iterative scheme can require only a few iterations to converge by eye. It is anticipated that the scheme will converge more slowly, if at all, for small reduced wavenumbers $L k_{0}$; indeed [20] proves convergence only for asymptotically large $L k_{0}$. Physical grounds for expecting the method to still work at low reduced frequencies may be considered as follows: despite the coupling becoming stronger as the junctions become nearer on the length scale of the acoustic field, the field scattered from a junction $A$ to another $B$ and back to $A$ should be weaker than the initial field that was scattered from $A$. Figure $2 b$ demonstrates that in fact further to previous work, the approach converges even at low frequencies, albeit more slowly. Undertaking the larger number of factorizations required is facilitated by the numerical implementation that enables an arbitrary number of iterations given a defined pattern in which to consider the scalar problems associated with the system.

Scattering of a convected gust We may also consider the scattering of a convective gust by a finite plate in a background mean flow. Figure 3 presents directivity patterns for the scattering of convected gust from a finite plate, varying the Mach number associated with the background flow and boundary condition imposed on the plate. For the frequency considered, increasing the Mach number increases the far-field noise, whilst imposing an acoustically softer boundary condition (perhaps mimicking porosity) reduces the far-field sound.

Discussion We briefly discuss connections with existing analytic approaches to consider scattering from a finite plate in the aeroacoustic community, namely Amiet's model [26] and its extensions including backscattering [24, 27, 28]. Amiet considers Schwarzschild's solution for scattering from a semi-infinite plane that is only forced on a finite section to mimic a flat plate of finite chord for a study of trailing edge noise. Roger and Moreau [27] introduce a leading edge correction to correct for flow upstream of the aerofoil, by solving a half-plane problem associated with the leading edge using Schwarzschild's solution. Further corrections may be included by iteration. Such an approach can solve the same set of problems as our iterative scheme. The crucial difference is that our scheme exists in spectral space, that is solves 


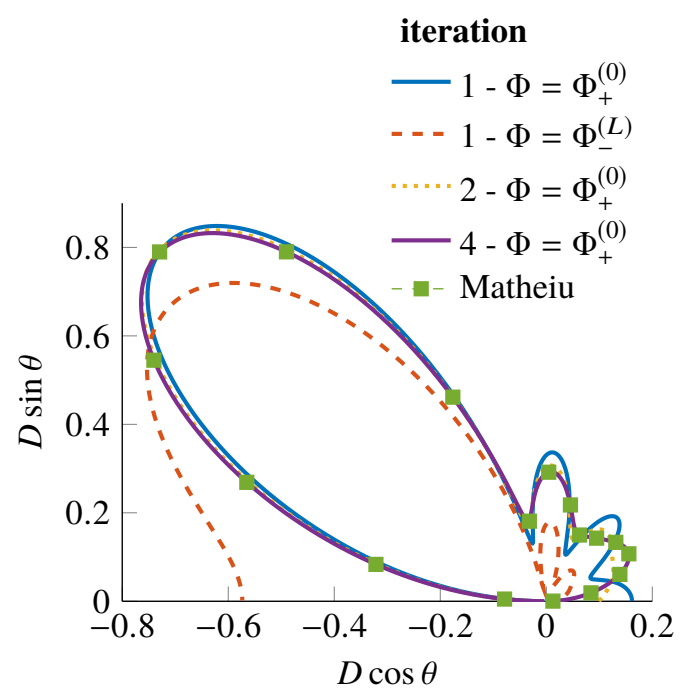

(a) Validation with Mathieu function solution.

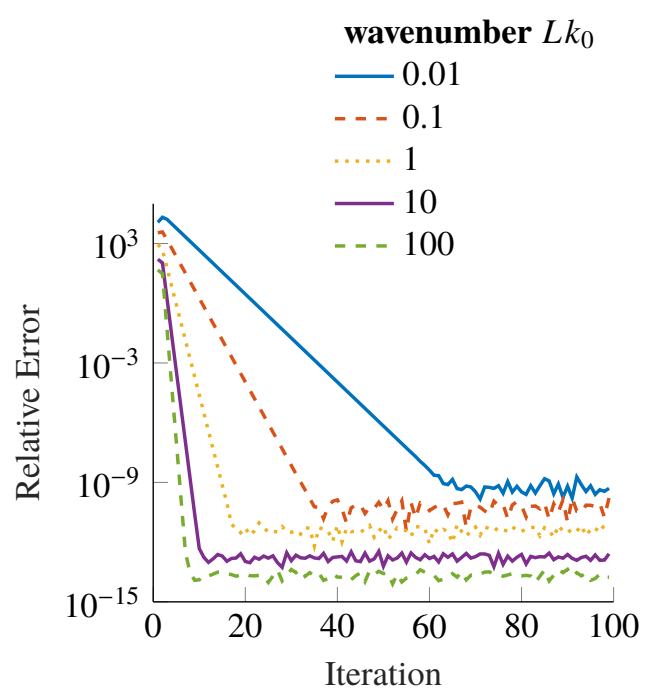

(b) Convergence against final iterate.

Fig. 2 Results for far-field directivity for scattering from a single plate of a plane wave incident at an angle $\theta_{i}=\pi / 4$ to the $x$ axis. (a) Comparison of far-field directivity for an incident plane wave with $L k_{0}=12$ and of our implementation with Matheiu function expansion derived for [11]. Legend indicates the iteration, and the manner in which $\Phi$ was recovered from the functions solved for in the iterative formulation. (b) Legend indicates the reduced wavenumber $L k_{0}$. The scheme converges exponentially for a range of frequencies. We observe faster convergence for larger reduced wavenumbers.

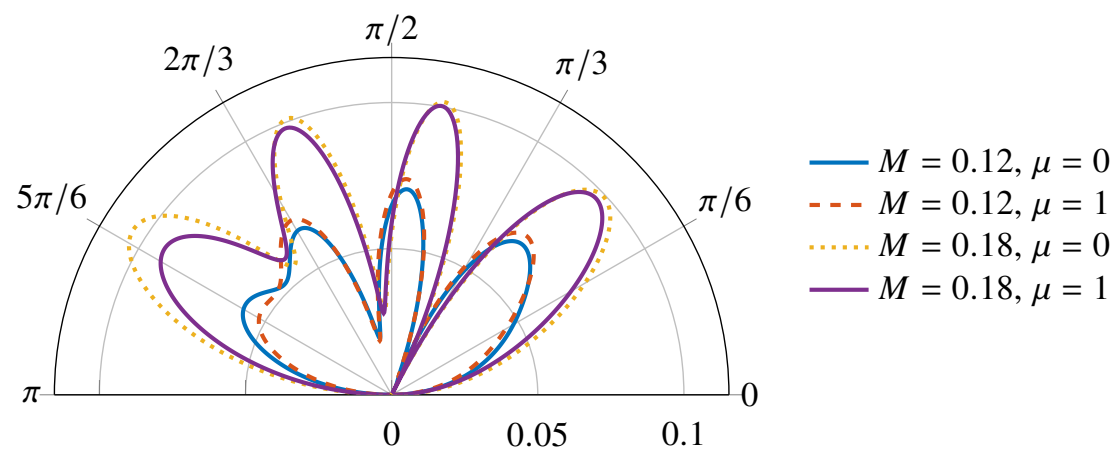

Fig. 3 Far field directivity for a convected gust of frequency $2000 \mathrm{~Hz}$ scattered from a finite plate of length $150 \mathrm{~mm}$, varying Mach number $M$ associated with the background flow and the impedance boundary condition on the plate described by $\mu$ 
for the variable $\alpha$. Firstly this enables well known expressions from steepest descent approximations for the far-field to be quickly applied, and secondly fits into the broader Wiener-Hopf literature allowing for established techniques to be incorporated to deal with problems involving alternative boundary conditions and governing equations.

\section{B. Many collinear finite plates}

The formulation for a single finite plate may be readily extended to many collinear finite plates. A schematic diagram is presented in figure 4. For rigid, impenetrable equations at $\{(x, 0)$ for $x \in \Gamma$ we now impose boundary conditions

incident wave $\phi_{i}$

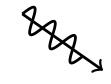

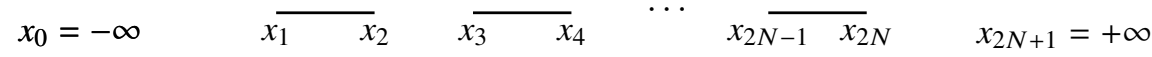

Fig. 4 Schematic diagram for scattering by a set of $N$ collinear finite plates

$$
\begin{aligned}
\phi^{\prime}(x, 0 \pm) & =q(x) \quad x \in \Gamma \\
{[\phi(x, 0)]_{-}^{+}=\left[\phi^{\prime}(x, 0)\right]_{-}^{+} } & =0 \quad x \in \mathbb{R} \backslash \Gamma
\end{aligned}
$$

together with the usual continuity of $\phi^{\prime}$. For two plates at $x \in[1,2]$ and $x \in[3,4]$ we find a spectral equation of the form

$$
\Phi_{[-, 1]}^{\prime}+Q_{2}+\Phi_{[2,3]}^{\prime}+Q_{4}+\Phi_{[4,+]}^{\prime}+\gamma Q_{1}+\gamma \Phi_{[1,2]}+\gamma Q_{3}+\gamma \Phi_{[3,4]}+\gamma Q_{5}=0
$$

In an analogous manner to the case for a single finite plate equation (22) may be formulated as a set of scalar problems associated with each junction. For instance, the explicit equation associated with the junction at $x=1$ is

$$
\Phi_{-}^{\prime(1)}+\gamma \Phi_{+}^{(1)}=-F^{(1)}-e^{i \alpha} \Phi_{+}^{\prime(2)}-e^{3 i \alpha} \Phi_{+}^{(4)}-e^{2 i \alpha} \gamma \Phi_{+}^{(3)},
$$

where $F^{(1)}=-Q_{2}-Q_{4}-\gamma Q_{1}-\gamma Q_{3}-\gamma Q_{5}$.

We note that alternative approaches to similar problems include hybrid-numerical asymptotics [29] and a sophisticated singular integral equation approach [21]. The method here is best suited to high frequencies and for the computation of far-field directivity due to an easy application of the steepest descent approximation.

Convergence We first consider the performance of the scheme as for larger Wiener-Hopf systems. In section II.B we noted that we must choose the order in which to navigate the system of scalar equations. This can have a dramatic effect upon the performance of the scheme. In particular, for larger numbers of plates conducting a forward then backward pass through the system is much more effective than only passing through the system forwards. This may be considered to correspond to correcting to the term most affected by the previous changes at each stage: updating the Fourier transform of the nearest boundary section.

Figure 5 presents the number of iterations required to achieve convergence to 5 significant figures (s.f.) of accuracy in the associated far-field directivity for various numbers of unit spaced, unit length rigid plates for different frequencies. An iteration is considered to comprise solving a scalar Wiener-Hopf problem for each junction. Convergence is fastest at high frequencies, taking a consistent number of iterations so defined to achieve a given precision. At low frequencies many more iterations may be required, and the number of iterations for a given wavenumber increases with the number of plates. We note that for mid to high frequencies the time to achieve a given accuracy scales quadratically with the number of junctions $N$; the number of passes through the system remains constant, but each pass requires the solution of $N$ scalar WH equation, each of which has $O(N)$ terms to be factorized. 


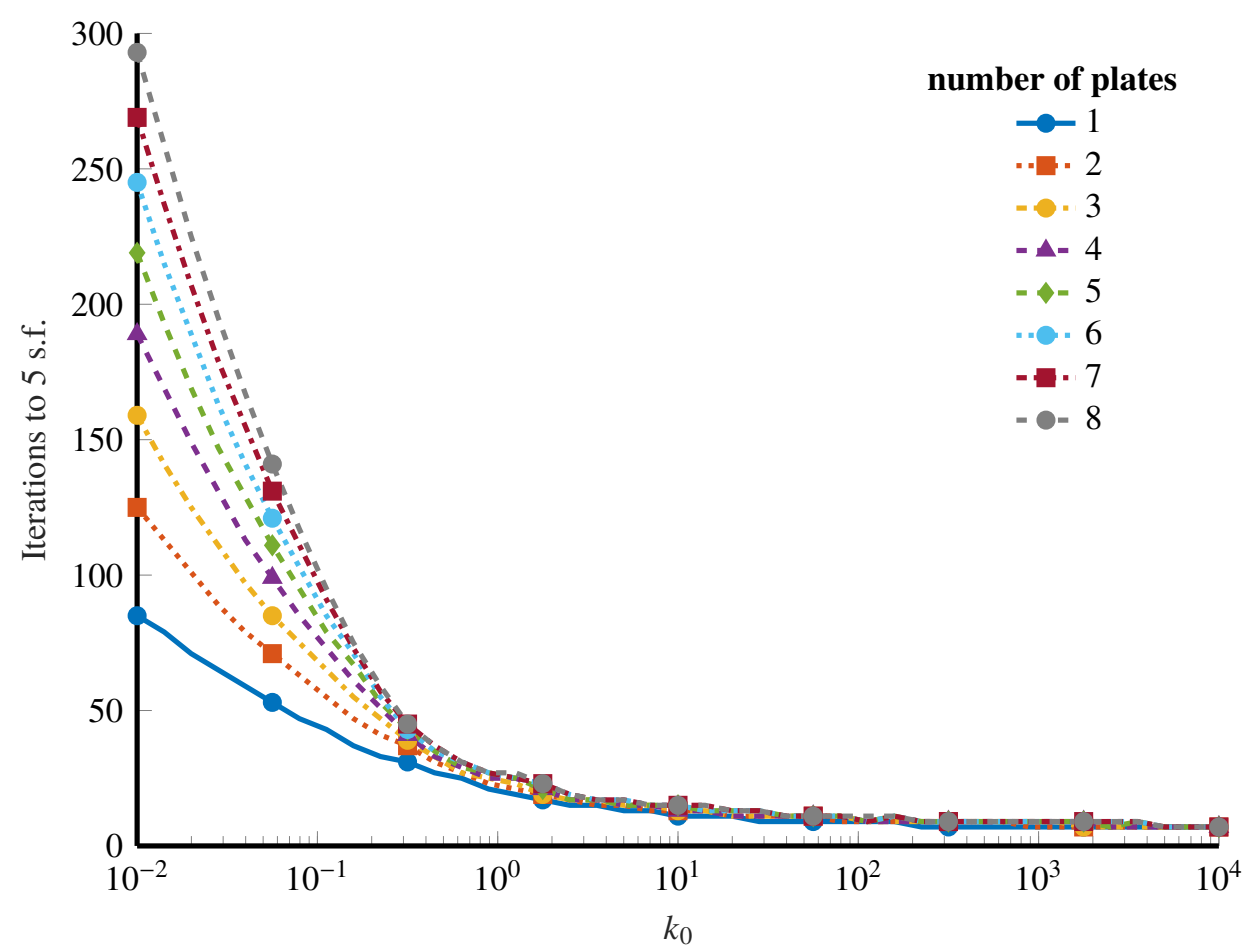

Fig. 5 Convergence of the iterative scheme for $N$ plates: Number of iterations required to reach a relative error of $10^{-5}$, varying the number of plates, as per legend; each of unit length and unit spacing.

Influence of a gap in a finite plate In scattering problems with multiple length scales it is useful to understand the validity of low frequency (acoustically compact) or high frequency (geometric optic) assumptions, especially as the interaction of diffracted fields can be difficult to anticipate. We now demonstrate how such phenomena may be investigated using the iterative Wiener-Hopf method to solve the precise and approximated problem.

We consider a plane wave incident on two collinear plates of equal length. When the gap between the plates is small and the wavelength of the incident wave is large it is natural to anticipate that the gap has a negligible effect upon the far-field. Similarly when the gap is large one would expect the two scattered fields to interact minimally, and so observe a far-field directivity that is simply twice that of a single plate.

In figure 6 we present results for two collinear plates varying gap size. For very small gap sizes the far-field directivity collapses onto that of a single plate of length $L=2$ (figure 6a). As the gap size is increased we observe far-field lobes saturating onto double the directivity pattern of the scattered field for a single finite plate of length $L=1$, that is as if the plates did not interact (figure 6b).

The present approach may so provide a useful theoretical tool to better understand pure acoustic scattering effects and so isolate other complex aeroacoustic phenomena present in physical systems.

\section{Future applications}

This section outlines some potential applications of this method and the associated theoretical differences. Since the method is based upon the solution of scalar Wiener-Hopf equations, any developments require the associated evaluation of the kernels at points of interest and their factorizations.

The problems so far considered are characterized by kernels involving branch cuts and exponential behaviour, and with anti-symmetry in the wall-normal direction. A first extension would be to break anti-symmetry, for instance by imposing distinct boundary conditions on the upper and lower surfaces of a plate or considering different media above and below a semi-infinite half-plane. A second extension would be to consider kernels involving poles. These arise in a finite number when considering an elastic boundary condition [9, 10], whereas for geometries requiring decomposition into sections of finite extent in the wall-normal direction, such as ducts, there are typically infinitely many[1, 7]. Two examples it may be interesting to investigate are: 
plate separation

$-10^{-5} \mathrm{~L}$

- *- 0 (no interaction)
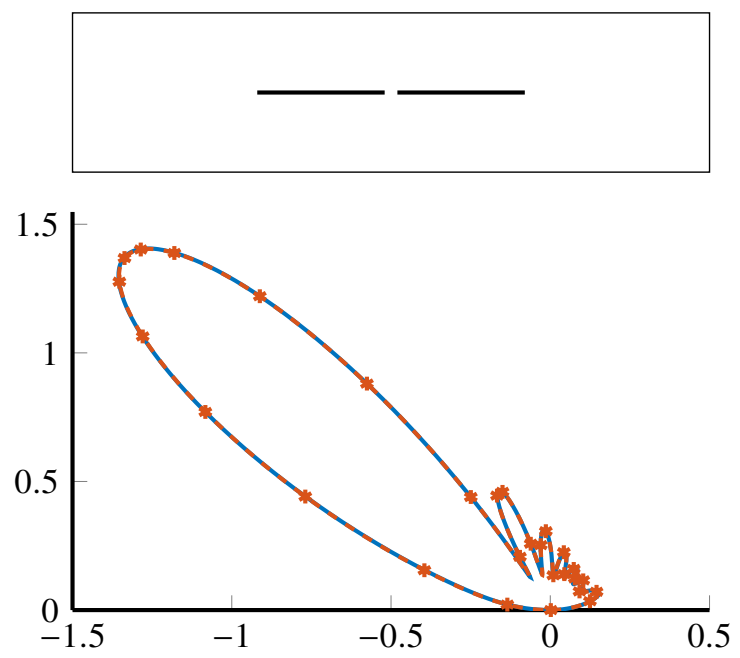

(a) Small gap: We observe a complex array of lobes with decreasing gap, eventually collapsing onto the no interaction case. The no interaction plot refers to a single plate of length $L=2$ i.e. no gap. plate separation

$-5 L$

$-=-\infty$ (no interaction)
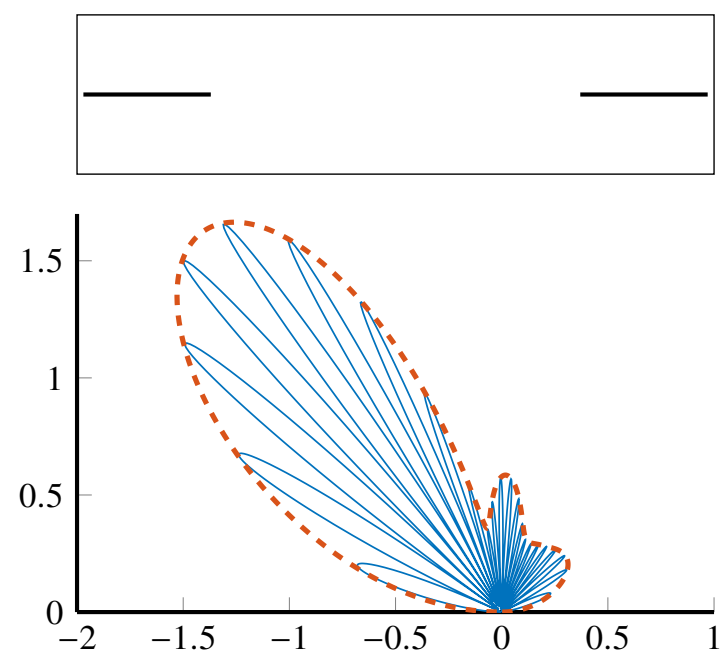

(b) Large gap: as the gap size is increased we find an increasing number of lobes, eventually saturating the no interaction case. The no interaction plot refers to twice the directivity of a single plate of length $L=1$ i.e. two plates neither aware of the others' existence.

Fig. 6 Two collinear plates of equal length $L=1$ and wavenumber $k_{0}=12$ 
- exhaust configurations for which the lining does not extend to the lip of the exhaust, but does not seem to have been considered in previous coaxial exhaust configuration studies using Wiener-Hopf, such as [7]

- canopies formed of one or many poroelastic plates above a rigid wall to provide a theoretical model for experimental designs [30]

Another direction would be to consider governing equations that yield kernels best treated numerically by integrating an ordinary differential equation in the complex plane, for instance that associated with sheared flow profiles [31]. It is less clear how far methods involving coordinate transforms could be incorporated, for instance to consider realistic aerofoil geometry [32] or spanwise serrations [33], as each transform will affect each boundary. However in simple cases, or by coupling the problems and iterating in the spatial domain, the present technique could provide a useful tool by which to extend such problems.

\section{Conclusion}

This paper demonstrates the utility of an iterative application of the Wiener-Hopf technique to investigate mixed boundary value problems of interest in aeroacoustics. The crucial extension enabled by this method is the solution of certain matrix Wiener-Hopf problems associated with problems involving multiple junctions at which the boundary condition imposed changes, rather than just one. Here we considered scattering from a finite plate of arbitrary porosity and scattering from an arbitrary number of collinear finite plates. The implementation employs an open source package, and automates much of the mathematical detail of the method, hopefully creating a practical tool for the engineering community. The speed and reliability of the method makes it ideal for theoretical parameter searches and optimisation studies to inform investigations of complex systems. This approach may be employed for more general linear differential operators in $2 \mathrm{D}$ and $3 \mathrm{D}$ domains with boundary conditions imposed on parallel sections. A significant extension would be to consider geometries that support modes; this could provide a useful tool to investigate scattering problems associated with lined duct intakes and exhausts, and bio-inspired canopies.

\section{Acknowledgments}

M. J. Priddin thanks S. Olver for advice regarding the use of SingularIntegralEquations.jl. A.V. Kisil acknowledges support from Sultan Qaboos Research Fellowship at Corpus Christi College at University of Cambridge. L. J. Ayton acknowledges support from EPSRC Early Career Fellowship EP/P015980/1.

\section{References}

[1] Noble, B., Methods based on the Wiener-Hopf technique for the solution of partial differential equations, Chelsea Pub Co, 1988.

[2] Crighton, D. G., and Leppington, F. G., "Scattering of aerodynamic noise by a semi-infinite compliant plate," Journal of Fluid Mechanics, Vol. 43, No. 04, 1970, p. 721. doi:10.1017/S0022112070002690.

[3] Barton, P. G., and Rawlins, A. D., "Diffraction by a half-plane in a moving fluid," The Quarterly Journal of Mechanics and Applied Mathematics, Vol. 58, No. 3, 2005, pp. 459-479. doi:10/fm5w8x.

[4] Munt, R. M., “The interaction of sound with a subsonic jet issuing from a semi-infinite cylindrical pipe,” Journal of Fluid Mechanics, Vol. 83, No. 4, 1977, pp. 609-640. doi:10/bh3pnr.

[5] Munt, R. M., "Acoustic transmission properties of a jet pipe with subsonic jet flow: I. The cold jet reflection coefficient," Journal of Sound and Vibration, Vol. 142, No. 3, 1990, pp. 413-436. doi:10/bb5hp5.

[6] Demir, A., and Rienstra, S. W., "Sound radiation from an annular duct with jet flow and a lined centerbody," Proceedings 12th AIAA/CEAS Aeroacoustics Conference (Cambridge MA, USA, May 8-10, 2006), Paper AIAA 2006-2718, 2006, pp. 1-18.

[7] Veitch, B., and Peake, N., "Acoustic propagation and scattering in the exhaust flow from coaxial cylinders," Journal of Fluid Mechanics, Vol. 613, 2008, pp. 275-307. doi:10/cnn24q.

[8] Demir, A., and Rienstra, S., "Sound Radiation from a Lined Exhaust Duct with Lined Afterbody," 16th AIAA/CEAS Aeroacoustics Conference, American Institute of Aeronautics and Astronautics, Stockholm, Sweden, 2010. doi:10.2514/6.2010-3947.

[9] Jaworski, J. W., and Peake, N., "Aerodynamic noise from a poroelastic edge with implications for the silent flight of owls," Journal of Fluid Mechanics, Vol. 723, 2013, pp. 456-479. doi:10/f4vkds. 
[10] Ayton, L. J., "Acoustic scattering by a finite rigid plate with a poroelastic extension," Journal of Fluid Mechanics, Vol. 791, 2016, pp. 414-438. doi:10/f8bvg3.

[11] Kisil, A., and Ayton, L. J., "Aerodynamic noise from rigid trailing edges with finite porous extensions," Journal of Fluid Mechanics, Vol. 836, 2018, pp. 117-144. doi:10/gcpv8k.

[12] Ayton, L. J., and Kim, J. W., "An analytic solution for the noise generated by gust-aerofoil interaction for plates with serrated leading edges," Journal of Fluid Mechanics, Vol. 853, 2018, pp. 515-536. doi:10/gd55sz.

[13] Ayton, L. J., "Analytic solution for aerodynamic noise generated by plates with spanwise-varying trailing edges," Journal of Fluid Mechanics, Vol. 849, 2018, pp. 448-466.

[14] Abrahams, I. D., "The application of Pade approximants to Wiener-Hopf factorization," IMA Journal of Applied Mathematics, Vol. 65, No. 3, 2000, pp. 257-281. doi:10/fvj5fk.

[15] Veitch, B. H., and David Abrahams, I., "On the commutative factorization of $n \times n$ matrix Wiener-Hopf kernels with distinct eigenvalues," Proceedings of the Royal Society A: Mathematical, Physical and Engineering Sciences, Vol. 463, No. 2078, 2007, pp. 613-639. doi:10/crv5v9.

[16] Crighton, D. G., “Asymptotic factorization of Wiener-Hopf kernels,” Wave Motion, Vol. 33, No. 1, 2001 , pp. 51-65. doi:10/crwsdr.

[17] Mishuris, G., and Rogosin, S., "An asymptotic method of factorization of a class of matrix functions," Proceedings of the Royal Society A: Mathematical, Physical and Engineering Sciences, Vol. 470, No. 2166, 2014, pp. $20140109-20140109$. doi: $10 / \mathrm{gd} 8 \mathrm{cq} 4$.

[18] Daniele, V., and Lombardi, G., "Fredholm factorization of Wiener-Hopf scalar and matrix kernels," Radio Science, Vol. 42, No. 6, 2007. doi:10.1029/2007RS003673.

[19] Trogdon, T., and Olver, S., Riemann-Hilbert Problems, Their Numerical Solution, and the Computation of Nonlinear Special Functions, SIAM, 2016.

[20] Kisil, A. V., "An Iterative Wiener-Hopf Method for Triangular Matrix Functions with Exponential Factors," SIAM Journal on Applied Mathematics, Vol. 78, No. 1, 2018, pp. 45-62. doi:10/gcp3w2.

[21] Slevinsky, R. M., and Olver, S., "A fast and well-conditioned spectral method for singular integral equations," Journal of Computational Physics, Vol. 332, 2017, pp. 290-315. doi:10/f9qr27.

[22] Glegg, S., and Devenport, W., Aeroacoustics of Low Mach Number Flows: Fundamentals, Analysis, and Measurement, Academic Press, 2017.

[23] McLachlan, N., Theory and Application of Mathieu Functions, Clarendon Press, 1947.

[24] Nigro, D., "Prediction of Broadband and hydrodynamic noise: derivation of analytical models for low frequency," Ph.D. thesis, University of Manchester, 2017.

[25] Colbrook, M. J., Ayton, L. J., and Fokas, A. S., "The unified transform for mixed boundary condition problems in unbounded domains," Proceedings of the Royal Society A: Mathematical, Physical and Engineering Sciences, Vol. 475, No. 2222, 2019, p. 20180605. doi:10.1098/rspa.2018.0605.

[26] Amiet, R. K., "Noise due to turbulent flow past a trailing edge," Journal of sound and vibration, Vol. 47, No. 3, 1976, pp. 387-393.

[27] Roger, M., and Moreau, S., "Back-scattering correction and further extensions of Amiet's trailing-edge noise model. Part 1: theory," Journal of Sound and Vibration, Vol. 286, No. 3, 2005, pp. 477-506. doi:10.1016/j.jsv.2004.10.054.

[28] Santana, L. D., Schram, C., and Desmet, W., "Low-frequency extension of Amiet's theory for compact airfoil noise predictions," Journal of Sound and Vibration, Vol. 372, 2016, pp. 342-356. doi:10/gc3hnb.

[29] Hewett, D. P., Langdon, S., and Chandler-Wilde, S. N., "A frequency-independent boundary element method for scattering by two-dimensional screens and apertures," IMA Journal of Numerical Analysis, Vol. 35, No. 4, 2015, pp. 1698-1728. doi:10.1093/imanum/dru043.

[30] Clark, I. A., Daly, C. A., Devenport, W., Alexander, W. N., Peake, N., Jaworski, J. W., and Glegg, S., "Bio-inspired canopies for the reduction of roughness noise," Journal of Sound and Vibration, Vol. 385, 2016, pp. 33-54. doi:10/f88zhx. 
[31] Schuster, W., "Trailing edge noise produced by the scattering of boundary layer turbulence," Ph.D. thesis, The University of Arizona, 2002.

[32] Ayton, L. J., "Asymptotic approximations for the sound generated by aerofoils in unsteady subsonic flows," Thesis, University of Cambridge, Oct. 2014. doi:10.17863/CAM.16135.

[33] Ayton, L. J., "Analytic solution for aerodynamic noise generated by plates with spanwise-varying trailing edges," Journal of Fluid Mechanics, Vol. 849, 2018, pp. 448-466. doi:10/gds7fp. 